Nervenarzt 2019 $\cdot 90: 361-370$

https://doi.org/10.1007/s00115-018-0630-1

Online publiziert: 15 . Oktober 2018

(c) Der/die Autor(en) 2018

CrossMark

K. Fheodoroff' $\cdot$ D. Dressler ${ }^{2} \cdot$ H. Woldag ${ }^{3} \cdot$ P. Koßmehl ${ }^{4} \cdot$ M. Koch $^{5} \cdot$ P. Maisonobe ${ }^{6} \cdot$

G. Reichel ${ }^{7}$

${ }^{1}$ Gailtal-Klinik, Hermagor, Österreich

${ }^{2}$ Medizinische Hochschule Hannover, Hannover, Deutschland

${ }^{3}$ Praxis Dr. Schäker, Leipzig, Deutschland

${ }^{4}$ Kliniken Beelitz GmbH, Beelitz-Heilstätten, Beelitz, Deutschland

${ }^{5}$ Ipsen Pharma, Ettlingen, Deutschland

${ }^{6}$ Ipsen, Les Ulis, Frankreich

${ }^{7}$ Paracelcus-Klinik Zwickau, Zwickau, Deutschland

\title{
Therapieziele bei Patienten mit Armspastik nach Schlaganfall nach Injektion mit Botulinumtoxin A
}

\section{Ergebnisse der deutsch-österreichischen Subgruppe der ULIS-II-Studie}

Der Einsatz von Botulinumtoxin A (BoNT-A) bei erwachsenen Patienten mit Armspastik nach Schlaganfall ist inzwischen als gut verträgliche und effektive Behandlungsmethode etabliert. Das Upper Limb International Spasticity (ULIS)-Programm untersucht die aktuelle Behandlungspraxis bei Armspastik nach Schlaganfall mit BoNT-A. Um einen Überblick über die Therapiepraxis hinsichtlich der Zielsetzung und -erreichung im deutschsprachigen Raum zu erhalten, werden in diesem Beitrag die Ergebnisse der deutschen und österreichischen Kohorte gegenüber der Gesamtkohorte dargestellt und verglichen.

Die redaktionelle Betreuung des Manuskripts wurde von Dr. Petra Jöstingmeyer (med:unit $\mathrm{GmbH}$, Köln) übernommen und von Ipsen Pharma GmbH finanziell unterstützt.

Aus Gründen der besseren Lesbarkeit wird in diesem Beitrag überwiegend das generische Maskulinum verwendet. Dies impliziert immer beide Formen, schließt also die weibliche Form mit ein.
Spastische Bewegungsstörungen sind eine häufige Folgeerscheinung nach einem Schlaganfall. Ursache ist eine Schädigung des ersten motorischen Neurons, wobei Spastizität als Anpassung an eine Läsion deszendierender motorischer Bahnen verstanden wird [11]. Die oberen Gliedmaßen sind häufiger betroffen [31]. Die Armspastik gilt als chronische und komplexe neurologische Erkrankung mit vielen möglichen Symptomen wie gesteigerten Reflexen, Kokontraktion, Muskelumbau und Dystonien. Aufgrund der Läsion im Zentralnervensystem bestehen häufig zusätzlich sensorische Störungen, Wahrnehmungsstörungen und kognitive Defizite.

Gemäß mehreren internationalen Konsensusempfehlungen [13, 21, 22] und nationalen Leitlinien [10] werden BoNT-A-Injektionen zur Behandlung der fokalen Spastizität nach Schlaganfall empfohlen. BoNT-A blockiert die cholinerge Übertragung an der motorischen Endplatte durch eine Unterbindung der Acetylcholinfreisetzung [8]. Der tonussenkende Effekt der Therapie wurde in mehreren klinischen Studien nachgewiesen $[4,5,7,23]$. In diesen Studien wurden in der Regel Veränderungen im Muskeltonus erfasst, jedoch nicht die Auswirkung auf die individuelle Behinderung. Da der Effekt der Spastizität auf die Motorik individuell unterschiedlich ist, können Patienten mit gleicher Diagnose und Schweregrad unterschiedliche Therapieziele haben. Die Methode der Zielerreichungsskalierung („Goal Attainment Scale“, GAS) ermöglicht eine statistische Analyse und Auswertung individualisierter Therapieziele [18, 25].

Aus klinischen Studien ist bekannt, dass die Behandlungspraxis mit BoNTA uneinheitlich ist, wobei Injektionstechniken, Ort der Injektion und Begleittherapien zentrumsabhängig variieren [2]. Um einen Überblick über den Einsatz von BoNT-A im Praxisalltag zu erhalten, wurde das Upper Limb International Spasticity (ULIS) -Programm gestartet. Es besteht aus mehreren internationalen Beobachtungsstudien zur aktuellen Behandlungspraxis der Armspastik mit BoNT-A [29]. ULIS-I war eine internationale Querschnittsstudie, mit der Daten zur klinischen Praxis im Hinblick auf Injektion, Dokumentation und Erfassung des Behandlungsergebnisses auf 


\section{Tab. 1 Studienablauf}

\begin{tabular}{llll}
\hline Maßnahmen & $\begin{array}{l}\text { Visite 1 } \\
\text { Studieneinschluss }\end{array}$ & $\begin{array}{l}\text { Visite 2 } \\
\text { Studienende }\end{array}$ \\
\hline Demografische Daten & $\mathrm{X}$ & - \\
\hline Anamnese & $\mathrm{X}$ & - \\
\hline Vorbehandlung/Begleittherapie für Armspastik & $\mathrm{X}$ & $\mathrm{X}$ \\
\hline Klinische Untersuchung & $\mathrm{X}$ & $\mathrm{X}$ \\
\hline Ziele definieren & $\mathrm{X}$ & - \\
\hline Injektion von BoNT-A & $\mathrm{X}$ & - \\
\hline Zielerreichung überprüfen (einschließlich GAS) & - & $\mathrm{X}$ \\
\hline Globale Einschätzung des Therapienutzens & - & $\mathrm{X}$ \\
\hline Therapiebedingte unerwünschte Ereignisse & - & $\mathrm{X}$ \\
\hline Weitere therapeutische Maßnahmen & - & $\mathrm{X}$ \\
\hline BoNT-A Botulinumtoxin A, GAS „Goal Attainment Scale“ & & \\
\hline
\end{tabular}

vier Kontinenten erhoben wurden [6]. Die noch laufende ULIS-III-Studie dokumentiert die Auswirkung eines integrierten Managements der Armspastik auf die Erreichung von Patientenzielen [26]. ULIS-II ist eine große, prospektive Verlaufsstudie, bei der die Responderrate anhand des mit GAS erfassten Grades der primären Zielerreichung nach einem Zyklus BoNT-A unter Alltagsbedingungen ermittelt wurde [28]. Insgesamt nahmen 456 Patienten aus 22 Nationen teil [29]. Hier stellen wir die Ergebnisse der Behandlungspraxis in Deutschland und Österreich im Vergleich zur Gesamtkohorte dar.

\section{Methodik}

\section{Studiendesign}

An der vorliegenden offenen, prospektiven, multizentrischen Beobachtungsstudie im Zeitraum von Januar 2010 bis Mai 2011 beteiligten sich weltweit 84 Zentren in 22 Ländern. Die Studie wurde mit Zustimmung der jeweils zuständigen Ethikkommission im Einklang mit dem jeweiligen nationalen Recht, den Leitlinien und Empfehlungen zur Sicherung guter epidemiologischer Praxis (GEP) sowie gemäß der Deklaration von Helsinki von 1975 (in der aktuellen, überarbeiteten Fassung) durchgeführt. Von allen beteiligten Patienten liegt eine Einverständniserklärung vor. Die Entscheidung des Arztes zur Behandlung mit BoNTA wurde unabhängig von der Teilnahme an der Studie getroffen. Die Behand- lung, einschließlich Begleittherapien und Verlaufskontrollen, folgte ausschließlich gemäß der üblichen ärztlichen Behandlungspraxis.

\section{Studienpopulation}

In die Studie aufgenommen wurden Patienten in einem Alter von $\geq 18$ Jahren mit einer Armspastik nach Schlaganfall, bei denen die Entscheidung für eine Behandlung mit BoNT-A bereits getroffen war, die letzte BoNT-A-Injektion mindestens 12 Wochen zurücklag sowie ein Einvernehmen über die Behandlungsziele hergestellt werden konnte, wobei diese Ziele möglichst nach einem Injektionszyklus erreichbar sein mussten.

\section{Studienablauf}

Insgesamt wurden weltweit 468 Patienten in die Studie eingeschlossen. Davon wurden 456 in der Wirksamkeitsanalyse berücksichtigt. Die prospektive Datenerhebung erfolgte über einen Zeitraum von maximal 5 Monaten mit 2 Beobachtungsterminen (Eingangsdokumentation vor Injektion mit BoNT-A, Abschlussdokumentation nach ca. 3 bis 5 Monaten). Um einen Zentrumseffekt zu vermeiden, war die Patientenzahl pro Zentrum auf 12 begrenzt.

\section{Endpunkte}

Primäres Zielkriterium zur Analyse der Wirksamkeit von BoNT-A war die Responderrate, definiert durch das Errei- chen des primären Ziels (GAS-Score 0, 1 oder 2) nach einem Zyklus BoNT-A entsprechend der Routinepraxis. Sekundäre Endpunkte waren demografische und erkrankungsspezifische Daten, Veränderungen der Körperfunktionsstörung, Erreichen von sekundären Zielen und des GAS-T-Score, globale Einschätzung des Therapienutzens, Injektionstechniken und Begleittherapien. - Tab. 1 gibt einen Überblick über den Studienablauf.

\section{Ziele setzen und messen (GAS)}

$\mathrm{Zu}$ Studienbeginn wurden im Gespräch mit dem Patienten die wesentlichen Problemfelder identifiziert. Zusammen mit dem Patienten wurden Ziele vereinbart (1 Hauptziel, bis zu 3 sekundäre Ziele), die innerhalb eines Injektionszyklus erreicht werden können. Als Ausgangswert wurde jedes Ziel in der Regel mit einem Wert von -1 bewertet (Baseline; Visite 1). In Ausnahmefällen, falls keine Verschlechterung der spastizitätsassoziierten Symptome zu erwarten war, wurde die Ausgangssituation mit -2 bewertet. Bei Visite 2 (nach einem Injektionszyklus) wurde der Grad der Zielerreichung vom Behandler gemeinsam mit dem Patienten verbal auf einer 6-stufigen Skala bewertet [25] und danach auf eine 5-stufige numerische Skala (von -2 bis +2 ) übertragen (•Tab. 2). Zur Berechnung des GAS-T-Scores wird eine standardisierte Formel verwendet [18], in der die Zielerreichungswerte numerisch (von -2 bis +2 ) sowie die Gewichtung der einzelnen Ziele (weniger wichtig, moderat wichtig, sehr wichtig) numerisch $(1,2$, 3) eingesetzt werden (๑Tab.2).

\section{Statistische Auswertung}

Die Auswertung der Daten erfolgte gemäß dem im Studienprotokoll festgelegten Analyseplan mittels SAS (Version 9.2, SAS Institute Inc., Cary, NC, USA). Zur Darstellung von Ausgangswerten und Wirksamkeitsanalysen wurden deskriptive statistische Verfahren einschließlich Berechnung des 95\%-Konfidenzintervalls $(\mathrm{KI})$ angewendet. Für die quantitativ skalierten Variablen wurden die folgenden statistischen Kennwerte bestimmt: Mittelwert, Standardabweichung, Me- 
dian, 1. Quartil sowie 3. Quartil. Die statistische Analyse zur Ermittlung des $p$ Werts des GAS-T-Scores nach Behandlung im Vergleich zur Baseline wurde mittels Signed-rank-Test durchgeführt.

Hier werden die Daten der D/A-Subgruppe im Vergleich mit der Gesamtpopulation als Post-hoc-Analyse dargestellt.

\section{Ergebnisse}

\section{Patientencharakteristika}

Insgesamt wurden im Rahmen der ULISII-Studie 45 Patienten in Deutschland und 14 Patienten in Österreich eingeschlossen und mit BoNT-A behandelt. Zwei Patienten schieden vorzeitig aus der Studie aus. Somit wurden 57 Patienten in der Wirksamkeitsanalyse und 59 Patienten in der Sicherheitsanalyse berücksichtigt. Demografische und Baseline-Daten sind in - Tab. 3 zusammengefasst. Hinsichtlich demografischer Daten waren die D/A-Patienten mit der Gesamtpopulation vergleichbar. Bei den D/A-Patienten lag der Schlaganfall im Vergleich zur Gesamtpopulation jedoch schon deutlich länger zurück $(101,0 \pm 80,9$ Monate vs. $61,4 \pm 69,1$ Monate). Entsprechend war im Vergleich zur Gesamtkohorte ein größerer Anteil der Patienten bereits vorbehandelt worden (93,0\% vs. 67,3\%) und ein höherer Anteil an Patienten hatte fixierte Kontrakturen (38,6\% vs. $25,4 \%)$. Bei den D/A-Patienten erfolgte die erste Injektion mit BoNT-A im
Durchschnitt $( \pm \mathrm{SD})$ vor $54,8 \pm 37,2 \mathrm{Mo}-$ naten. Seitdem waren im Mittel $( \pm S D)$ $15,1 \pm 12,9$ Zyklen verabreicht worden. In der Gesamtkohorte waren es nur 6,4 \pm 8,0 Zyklen in 33 Monaten. Die letzte Injektion lag bei den D/A-Patienten durchschnittlich $( \pm S D)$ 6,0 $\pm 11,5$ Monate und in der Gesamtkohorte $8,0 \pm 11,5$ Monate zurück. Das Intervall zwischen den Injektionen betrug in der D/A-Subgruppe im Durchschnitt $( \pm S D) 6,1 \pm 11,4$ Monate bzw. 8,0 $\pm 10,9$ Monate für die Gesamtkohorte.

\section{Therapieziele}

Aus den für Patienten und Betreuer relevanten Kategorien (Körperfunktionsstörung, aktive und passive Funktion, Schmerz, Mobilität, unwillkürliche Bewegung) wurde die Körperfunktionsstörung als häufigstes primäres Ziel (- Abb. 1a) in der D/A-Gruppe genannt (33,3\%). In der Gesamtkohorte war dies mit 23,0\% nur das zweithäufigste Therapieziel hinter den Zielen mit Bezug $\mathrm{zu}$ passiven Funktionen (Handhygiene, Pflegeerleichterung, 29,0\%), die nur von 19,3\% der D/A-Patienten als primäres Ziel genannt wurden. In der Gesamtkohorte wurden auf aktive Funktionen bezogene Ziele (Greifen - Loslassen feinmotorischer Handgebrauch, 14,0\% vs. $22,8 \%$ ) häufiger angestrebt, während bei D/A-Patienten mehr Wert auf Ziele im Zusammenhang mit Schmerz $(21,1 \%$ vs. $13,4 \%)$ und Mobilität (Ver- besserung des Bewegungsmusters beim Gehen, insbesondere Armpendel; 10,5\% vs. 2,2\%) gelegt wurde. Die Häufigkeiten der Angaben zu den sekundären Zielen waren ähnlich verteilt (• Abb. 1b), jedoch nannten wesentlich mehr D/APatienten Mobilität als sekundäres Ziel im Vergleich zur Gesamtkohorte (14,0\% vs. $4,0 \%)$.

\section{Injektionspraxis}

Sowohl bei den D/A-Patienten als auch weltweit wurden im Durchschnitt 5 Muskeln mit BoNT-A injiziert. Die am häufigsten behandelten Muskeln bei den D/A-Patienten waren der Fingerbeuger Musculus flexor digitorum superficialis $(80,7 \%)$, gefolgt vom Musculus biceps brachii (64,9\%), dem tiefen Fingerbeuger Musculus flexor digitorum profundus $(63,2 \%)$, Musculus brachioradialis $(54,4 \%)$ und Musculus brachialis $(42,1 \%)$. Dagegen wurde BoNT$\mathrm{A}$ in der Gesamtkohorte am häufigsten in den Musculus flexor digitorum superficialis (71,3\%), Musculus biceps brachii $(59,2 \%)$, Musculus flexor digitorum profundus $(58,1 \%)$, Musculus flexor carpi radialis $(57,5 \%)$ und Musculus brachioradialis $(34,2 \%)$ injiziert. In Deutschland und Österreich wurde elektrische Stimulation (ES) häufiger (26,3\%) eingesetzt als Elektromyographie (EMG; 12,3\%). Beide Techniken kamen bei den D/A-Patienten seltener zum Einsatz als in der Gesamtkohorte

Hier steht eine Anzeige. 
Nervenarzt 2019·90:361-370 https://doi.org/10.1007/s00115-018-0630-1

(c) Der/die Autor(en) 2018

K. Fheodoroff · D. Dressler · H. Woldag · P. Koßmehl · M. Koch · P. Maisonobe · G. Reichel

\section{Therapieziele bei Patienten mit Armspastik nach Schlaganfall nach Injektion mit Botulinumtoxin A. Ergebnisse der deutsch-österreichischen Subgruppe der ULIS-II-Studie}

\section{Zusammenfassung}

Hintergrund. ULIS-II war eine internationale Studie (NCT01020500) zur Erfassung der aktuellen Behandlungspraxis von erwachsenen Patienten mit Armspastik nach Schlaganfall mit Botulinumtoxin A (BoNT-A).

Fragestellung. Post-hoc-Analyse der Behandlungspraxis bei Spastik nach Schlaganfall bezüglich Zielsetzung und -erreichung nach einmaliger Behandlung mit BoNT-A in Bezug auf mögliche Unterschiede zwischen Deutschland (D) und Österreich (A) im Vergleich zur Gesamtkohorte der ULIS-IIStudie.

Material und Methoden. ULIS-II wurde als offene, prospektive, multizentrische Beobachtungsstudie mit 2 Visiten in 84 Zentren weltweit an 468 Patienten $\geq 18$ Jahre mit Armspastik nach Schlaganfall durchgeführt. Primäres Zielkriterium war die Responderrate, definiert als das Erreichen eines "Goal Attainment Scale" (GAS)-Scores von 0, 1 oder 2 nach einem Zyklus BoNT-A.

Ergebnisse. Insgesamt 57 Patienten aus D/A wurden in die Wirksamkeitsanalyse aufgenommen. Die Anzahl Patienten aus D/A und dem Gesamtkollektiv mit erreichtem primärem $(78,9 \%$ vs. $79,6 \%)$ und sekundärem Therapieziel $(76,8 \%$ vs. $75,6 \%)$ war vergleichbar. Im Unterschied zur Gesamtkohorte wurden in D/A Körperfunktionsstörung als häufigstes primäres Ziel genannt (33,3\%). Nach 3 bis 5 Monaten waren weniger Begleittherapien erforderlich als zu Studienbeginn: Patienten mit oraler antispastischer Medikation $40,4 \%$ vs. $61,4 \%$, Lagerung $36,8 \%$ vs. 50,9\%, Schienen $31,6 \%$ vs. $43,9 \%$. Im D/A-Kollektiv kamen weniger Injektionskontrolltechniken zum
Einsatz als in der Gesamtstudienpopulation (elektrische Stimulation: $26,3 \%$ vs. $45,8 \%$, Elektromyographie: $12,3 \%$ vs. $29,2 \%$ ). In der D/A-Subgruppe wurden keine unerwünschten Ereignisse dokumentiert.

Schlussfolgerung. Nach einmaliger Injektion von BoNT-A bei erwachsenen Patienten mit Armspastik wurde eine hohe Responderrate von ca. $80 \%$ in beiden Kohorten erzielt. Die Injektion mit BoNT-A führte zu Verbesserungen im Lebensalltag von Patienten und Betreuern, die über die Reduktion von Muskeltonus oder Spastizität hinausgehen.

Schlüsselwörter

Botulinumtoxin A - Behandlungsziele .

Armspastik · Goal Attainment Scaling · ULIS

\section{Treatment goals in patients with post-stroke upper limb spasticity following injection of botulinum toxin A. Results of the German-Austrian subgroup of the ULIS-II study}

\section{Abstract}

Background. The ULIS-II was an international cohort study (NCT01020500) evaluating current treatment of upper limb spasticity in post-stroke adult patients with botulinum toxin A (BoNT-A) in real-life practice. Objective. Post hoc analysis to compare current management of post-stroke adult patients regarding goal setting and attainment with BoNT-A in Germany (D) and Austria (A) with the full cohort of ULIS-II.

Material and methods. The ULIS-II was a global, open-label, prospective, multicenter observational study with 2 visits conducted in 84 centers worldwide. A total of 468 patients aged $\geq 18$ years with post-stroke upper limb spasticity were included. The primary outcome measure was the responder rate defined as achievement of a goal attainment scale (GAS) score of 0,1 or 2 after 1 cycle of BoNT-A.

Results. A total of 57 patients from D/A were included in the efficacy analysis. The number of patients in D/A and the full cohort achieving the primary $(78.9 \%$ vs. $79.6 \%)$ and secondary treatment goal (76.8\% vs. $75.6 \%)$, respectively, was comparable. Deviating from the full cohort, the most common primary treatment goal in D/A was related to impairment (33.3\%). Compared to baseline there was a marked reduction in concomitant therapies at the follow-up visit after 3-5 months in the D/A group: patients receiving oral anti-spastic medication $61.4 \%$ vs. $40.4 \%$, positioning $50.9 \%$ vs. $36.8 \%$ and splinting $43.9 \%$ vs. $31.6 \%$. Injection control techniques were less frequently used in the D/A group compared to the global study cohort (electrical stimulation: $26.3 \%$ vs. $45.8 \%$ and electromyography: $12.3 \%$ vs. $29.2 \%$ ). No adverse events were documented in the D/A cohort.

Conclusion. A single injection of BoNT-A in adult patients with post-stroke spasticity of the arm led to a high response rate of approximately $80 \%$ in both cohorts. The BoNT$A$ injections in post-stroke adult patients contributed to an improvement in the daily life of patients and their carers beyond simple reduction of muscle tone or spasticity.

\section{Keywords}

Botulinum toxin A - Treatment goals - Upper limb spasticity · Goal-attainment scale · ULIS
(ES: 45,8\%, EMG: 29,2\%). Eine Wiederholung der Injektion mit BoNT-A war für $55 \mathrm{D} / \mathrm{A}$-Patienten $(96,5 \%)$ geplant.

\section{Wirksamkeit}

Die Anzahl D/A-Patienten, die ihr primäres Therapieziel erreicht haben, war vergleichbar mit der des Gesamtkollek- tivs (78,9\%; 95\%-KI: 66,1-88,6\% bzw. 79,6\%; 95\%-KI: 75,6-83,2\%; • Abb. 2a). Die Behandlung führte zu einem statistisch signifikanten Anstieg des GAS-TScore um $14,8 \pm 8,5$ von Visite 1 (Baseline) $\mathrm{zu}$ Visite $2(p<0,0001)$ nach einem Behandlungszyklus. Der mittlere gewichtete GAS-T-Score zu Visite 2 betrug bei D/A-Patienten 48,1 \pm 7,9 gegenüber
$52,0 \pm 10,1$ in der Gesamtkohorte. Die sekundären Ziele wurden zu 76,8 \% (95\%KI: $63,6-87,0 \%)$ bei den D/A-Patienten und zu 75,6\% (95\%-KI: 71,2-79,2\%) in der Gesamtkohorte erreicht (• Abb. 2b).

Zusätzlich zu Physiotherapie und Ergotherapie waren bei den D/A-Patienten zur 2. Visite wesentlich weniger Begleittherapien erforderlich als zu Studienbe- 
Tab. 2 Skalierung der GAS

\begin{tabular}{|c|c|c|c|c|c|c|c|c|}
\hline \multirow[b]{3}{*}{ Verbale Skala } & \multicolumn{2}{|c|}{ Baseline } & \multicolumn{6}{|c|}{ Nach einem Behandlungszyklus } \\
\hline & \multicolumn{2}{|c|}{$\begin{array}{l}\text { Gibt es in dem Ziel eine } \\
\text { Restfunktion? }\end{array}$} & \multicolumn{6}{|c|}{ Wurde das Behandlungsziel erreicht? } \\
\hline & $\mathrm{Ja}$ & Nein & $\begin{array}{l}\text { Schlechter } \\
\text { geworden }\end{array}$ & $\begin{array}{l}\text { Keine Verän- } \\
\text { derung }\end{array}$ & $\begin{array}{l}\text { Partiell verbes- } \\
\text { sert }\end{array}$ & $\begin{array}{l}\text { Wie erwar- } \\
\text { tet }\end{array}$ & $\begin{array}{l}\text { Etwas besser als } \\
\text { erwartet }\end{array}$ & $\begin{array}{l}\text { Viel besser } \\
\text { als erwartet }\end{array}$ \\
\hline \multirow{2}{*}{$\begin{array}{l}\text { Numerische } \\
\text { Skala }\end{array}$} & \multirow[t]{2}{*}{-1} & & -2 & \multicolumn{2}{|l|}{-1} & 0 & 1 & 2 \\
\hline & & -2 & \multicolumn{2}{|l|}{-2} & -1 & 0 & 1 & 2 \\
\hline \multicolumn{3}{|l|}{ Ziel erreicht? } & \multicolumn{3}{|l|}{ Nein } & \multicolumn{3}{|l|}{$\mathrm{Ja}$} \\
\hline \multicolumn{9}{|c|}{ Gewichtung des Ziels (optional) zur Berechnung des GAS-T-Scores } \\
\hline Verbale Skala & \multicolumn{2}{|c|}{$\begin{array}{l}\text { Nicht zutreffend } \\
\text { Wichtig/Zutreffend }\end{array}$} & \multicolumn{2}{|c|}{ Wenig wichtig } & \multicolumn{2}{|l|}{ Moderat wichtig } & \multicolumn{2}{|l|}{ Sehr wichtig } \\
\hline $\begin{array}{l}\text { Numerische } \\
\text { Skala }\end{array}$ & \multicolumn{2}{|l|}{0} & \multicolumn{2}{|l|}{1} & \multicolumn{2}{|l|}{2} & \multicolumn{2}{|l|}{3} \\
\hline
\end{tabular}

Tab. 3 Demografische und Baseline-Daten

\begin{tabular}{|c|c|c|}
\hline Parameter & $\begin{array}{l}\text { Deutsch-österreichische } \\
\text { Subgruppe }(n=57)\end{array}$ & $\begin{array}{l}\text { Gesamtkohorte } \\
(n=456)\end{array}$ \\
\hline Alter, $\mathrm{MW} \pm \mathrm{SD}$ & $58,3 \pm 11,1$ Jahre & $56,7 \pm 13,5$ Jahre \\
\hline \multicolumn{3}{|l|}{ Geschlecht, $n$ (\%) } \\
\hline Männlich & $35(61,4 \%)$ & $266(58,3 \%)$ \\
\hline Weiblich & $22(38,6 \%)$ & $190(41,7 \%)$ \\
\hline Anzahl Monate seit Schlaganfall (MW \pm SD) & $101,0 \pm 80,9$ Monate & $61,4 \pm 69,1$ Monate \\
\hline \multicolumn{3}{|l|}{ Ätiologie des Schlaganfalls, $n$ (\%) } \\
\hline Infarkt & $37(64,9 \%)$ & $320(70,2 \%)$ \\
\hline Hirnblutung & $20(35,1 \%)$ & $139(30,5 \%)$ \\
\hline Infarkt und Hirnblutung & 0 & $3(0,7 \%)$ \\
\hline \multicolumn{3}{|l|}{ Lokalisation des Schlaganfalls, $n$ (\%) } \\
\hline Linke Hirnhälfte & $27(47,4 \%)$ & $215(47,1 \%)$ \\
\hline Rechte Hirnhälfte & $29(50,9 \%)$ & $235(51,5 \%)$ \\
\hline Bilateral & 0 & $4(0,9 \%)$ \\
\hline Hinterer Hirnkreislauf & $1(1,8 \%)$ & $13(2,9 \%)$ \\
\hline \multicolumn{3}{|l|}{ Lokalisation der Spastik, n (\%) } \\
\hline Schulter & $20(35,1 \%)$ & $147(37,2 \%)$ \\
\hline Oberarm & $46(80,7 \%)$ & $336(73,7 \%)$ \\
\hline Unterarm & $54(94,7 \%)$ & $434(95,2 \%)$ \\
\hline Hand und Finger & $21(36,8 \%)$ & $204(44,7 \%)$ \\
\hline Fixierte Kontrakturen & $22(38,6 \%)$ & $116(25,4 \%)$ \\
\hline Schwerwiegende Schwächen & $35(61,4 \%)$ & $264(57,9 \%)$ \\
\hline Vortherapie mit BoNT-A n (\%) & $53(93,0 \%)$ & $307(67,3 \%)$ \\
\hline Erste Injektion von BoNT-A (MW \pm SD) & $54,8 \pm 37,2$ Monate & $33,2 \pm 30,7$ Monate \\
\hline Letzte Injektion von BoNT-A (MW \pm SD) & $6,0 \pm 11,5$ Monate & $8,0 \pm 11,5$ Monate \\
\hline Bisherige Anzahl Zyklen (MW \pm SD) & $15,1 \pm 12,9$ & $6,4 \pm 8,0$ \\
\hline Injektionsintervall (MW \pm SD) & $6,1 \pm 11,4$ Monate & $8,0 \pm 10,9$ Monate \\
\hline
\end{tabular}

ginn (• Abb. 3). Der Anteil an Patienten in der D/A-Gruppe mit oraler antispastischer Medikation verringerte sich von 61,4 auf 40,4\% (Gesamtkohorte: 46,1 auf $28,5 \%)$, die Notwendigkeit einer Lagerung reduzierte sich von 50,9 auf $36,8 \%$ (Gesamtkohorte: 58,3 auf 52,0\%) und der Bedarf an Schienen nahm von 43,9 auf $31,6 \%$ ab (Gesamtkohorte: 41,2 auf $32,5 \%)$.

In der D/A-Gruppe gab es geringe Unterschiede in der globalen Einschätzung des Therapienutzens zwischen Ärzten und Patienten (• Abb. 4). Die Ärzte sahen für $75,4 \%$ der Patienten einen Therapienutzen und für 22,8\% keine Veränderung (Gesamtkohorte: 90,1\% bzw. 9,0\%). Dagegen empfanden 80,7\% der Patienten einen Therapienutzen und $12,3 \%$ keine Veränderung (Gesamtkohorte: $85,7 \%$ bzw. 12,9\%).

\section{Verträglichkeit}

In der deutsch/österreichischen Subgruppe wurden keine therapiebedingten unerwünschten Ereignisse im Studienverlauf dokumentiert. In der Gesamtkohorte wurde ein unerwünschtes Ereignis mit Kausalzusammenhang zu BoNT-A (Armmuskelschwäche) berichtet.

\section{Diskussion}

In einer weltweit durchgeführten Beobachtungsstudie wurden Wirksamkeit und Sicherheit von BoNT-A bei der routinemäßigen Behandlung von 


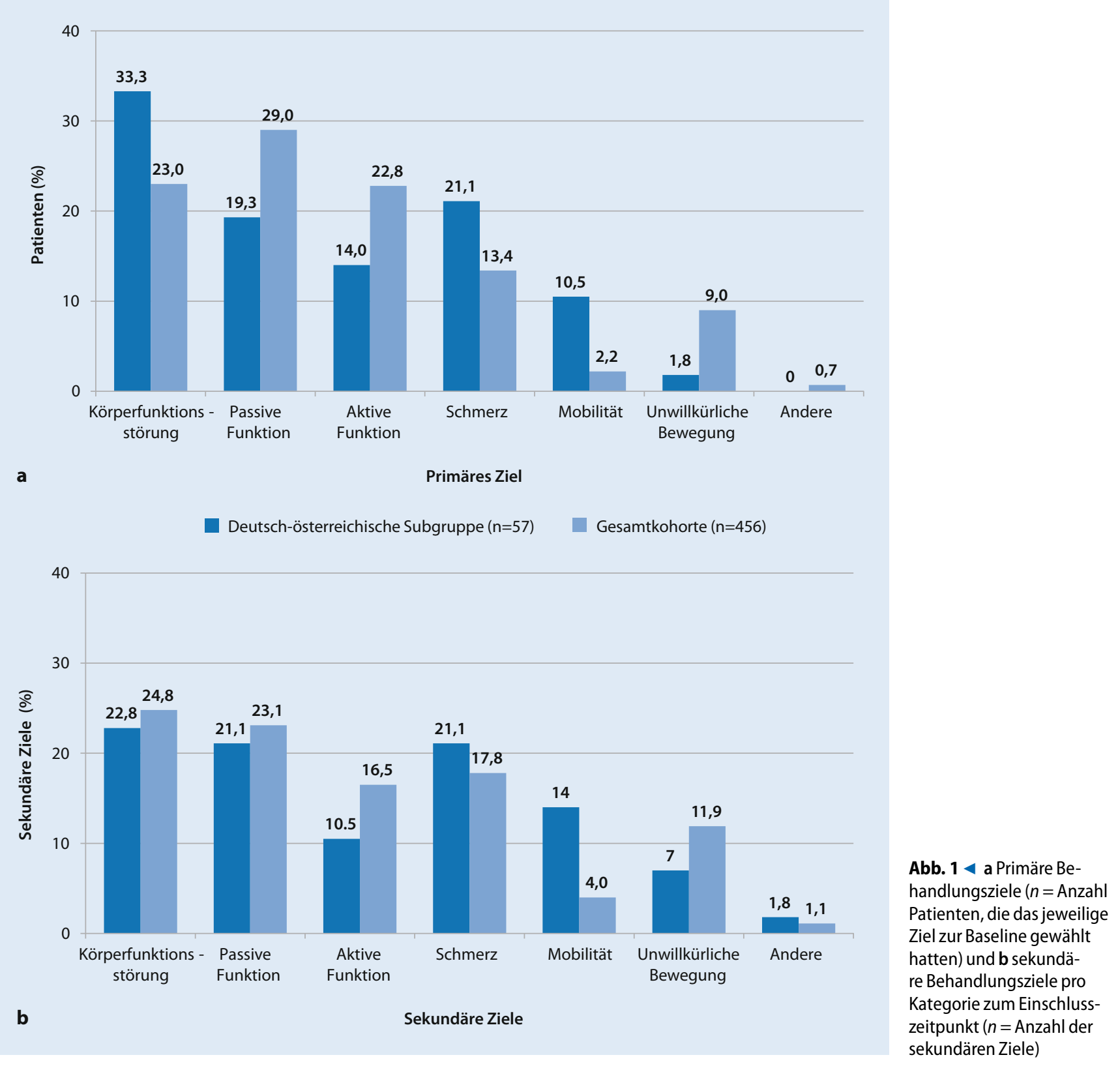

Armspastiken nach Schlaganfall untersucht [28, 29]. Um einen Überblick über das Therapiemanagement bezüglich der Zielsetzung und Zielerreichung im deutschsprachigen Raum zu erhalten, wurde die deutsch/österreichische Subgruppe post hoc analysiert und mit der Gesamtpopulation verglichen. In Übereinstimmung mit der Gesamtpopulation wurde mit ca. $80 \%$ eine hohe Responderrate erzielt, definiert als das Erreichen des primär gesetzten individuellen Therapieziels. Im Vergleich zur Gesamtkohorte waren die Patienten der D/A-Gruppe bereits länger mit BoNT-
A vorbehandelt, die Anzahl der vorangegangenen Behandlungszyklen sowie der Anteil der Patienten mit fixierten Kontrakturen waren höher. Dennoch führte die aktuelle Therapie zu einem neuerlichen Behandlungserfolg im Sinne der individuellen Zielerreichung. Dies stärkt die Rationale für eine Anwendung von BoNT-A bei chronischer Spastizität, insbesondere in Anbetracht der limitierten Behandlungsoptionen. Ebenfalls für die Eignung in der Langzeitanwendung spricht, dass bei mehrfacher Anwendung keine neutralisierenden Antikörper gefunden [3] und langfristige
Verbesserungen in aktiver und passiver Funktion beobachtet wurden [16, 19].

Die Verwendung von GAS als primäres Maß für den Therapieerfolg wird in der Literatur kontrovers diskutiert. Während einige Experten Bedenken in Bezug aufdie Validitätäußerten [24] und andere die Entwicklung standardisierter Zielbereiche anregten $[24,32]$, wird GAS zunehmend als sensible Methode zur Erfassung patientenbezogener Therapieergebnisse angesehen $[12,17]$. Besonders die große Bandbreite von Zielbereichen gilt als Vorteil dieser statistischen Methode [1, 9, 20, 27]. Unter Berücksichtigung der 


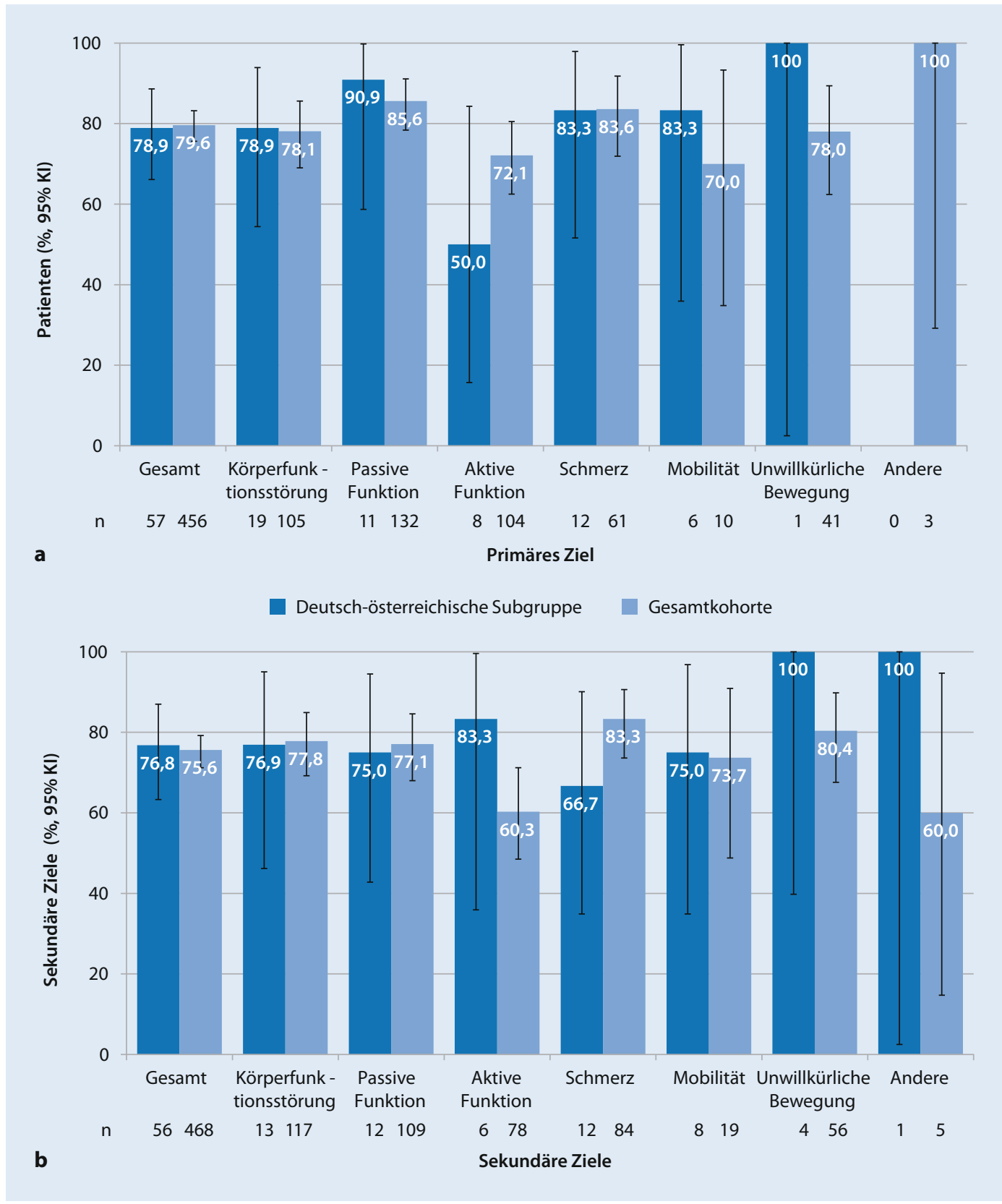

Abb. $2 \triangleleft$ Erreichung a der primärenZiele $(n=$ Anzahl Patienten, die das jeweilige Ziel zur Baseline gewählt hatten) und $\mathbf{b}$ der sekundären Ziele $(n=$ Anzahl der sekundären Ziele [ohne "unzutreffende“])

Limitierungen der GAS wurde die strukturiertere GAS-eous („Goal Attainment Scale-Evaluation of Upper Limb Spasticity“) für den klinischen Alltag entwickelt und wird in der aktuell laufenden ULIS-III-Studie eingesetzt [26]. Im Gegensatz zu standardisierten Bewertungsskalen entscheiden bei GAS und GASeous die Patienten, welches individuelle Therapieziel sie als wichtig erachten. Hierdurch gewinnt der Patient einen größeren Einfluss auf den Therapieerfolg. So lange es sich um spezifische, bewertbare und realistisch $\mathrm{zu}$ erreichende Ziele handelt, die der behandelnde Arzt zusammen mit dem Patienten vereinbart, besitzen alle genannten Ziele eine medizinische Relevanz.

Eine Post-hoc-Analyse der ULIS-IIStudiendaten ergab, dass auch bei Vorhandensein von Kontrakturen noch sinnvolle Behandlungsziele gesetzt und erreicht werden können. Das Erreichen der Therapieziele führte wiederum zu einer höheren Patientenzufriedenheit mit der Therapie. Dabei wurde die Auswahl des primären Ziels durch die Schwere der Einschränkung motorischer Funktionen,
Kontrakturen und Spastizität sowie Alter und Zeitpunkt des Schlaganfalls beeinflusst [14]. Ein Zusammenhang zwischen der Wahl des primären Ziels und der Erkrankungsdauer wurde auch bereits in einer internationalen Querschnittsstudie bestätigt [6]. Bei Patienten mit einer Erkrankungsdauer von $<3$ Monaten standen Ziele mit Bezug zu aktiven Funktionen im Vordergrund. Bei chronischen Patienten waren es dagegen Ziele mit Bezug zu passiven Funktionen, wie Erleichterung der Unterstützung beim Ankleiden, Körperhygiene und Erhalt der Be- 


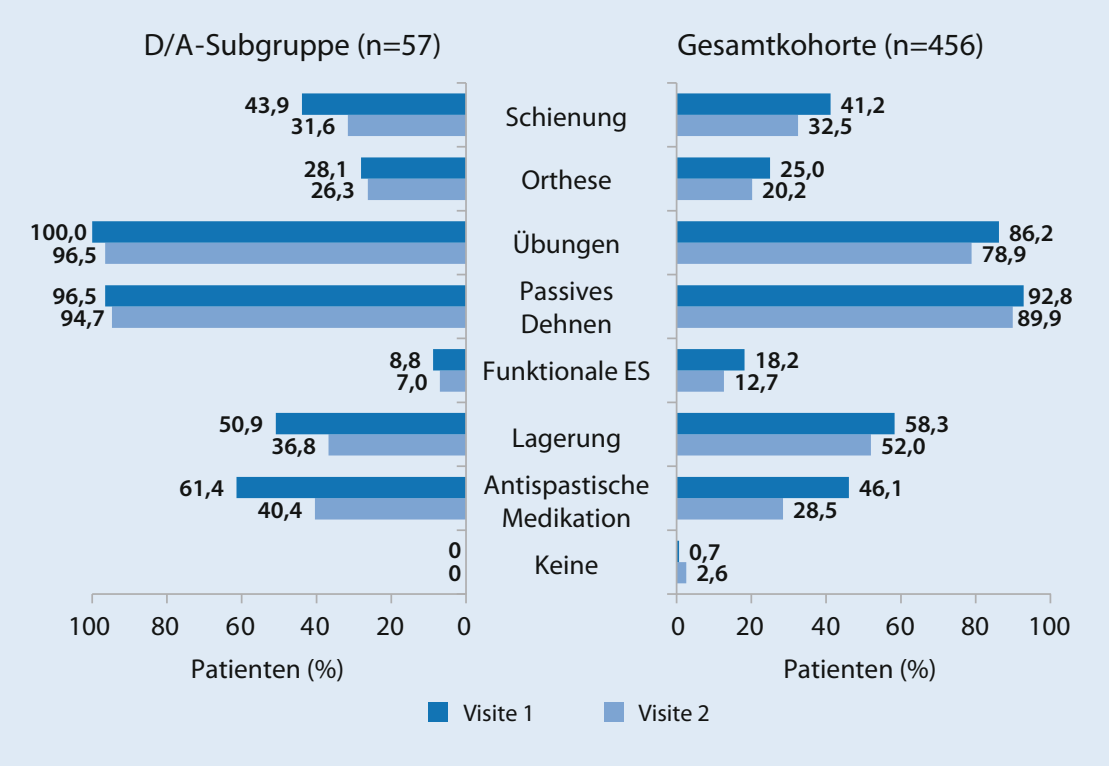

Abb. 3 A Vergleich der erforderlichen Begleittherapien zur 1. und 2. Visite in der deutsch-österreichischen Patientengruppe und der Gesamtkohorte. ES elektrische Stimulation

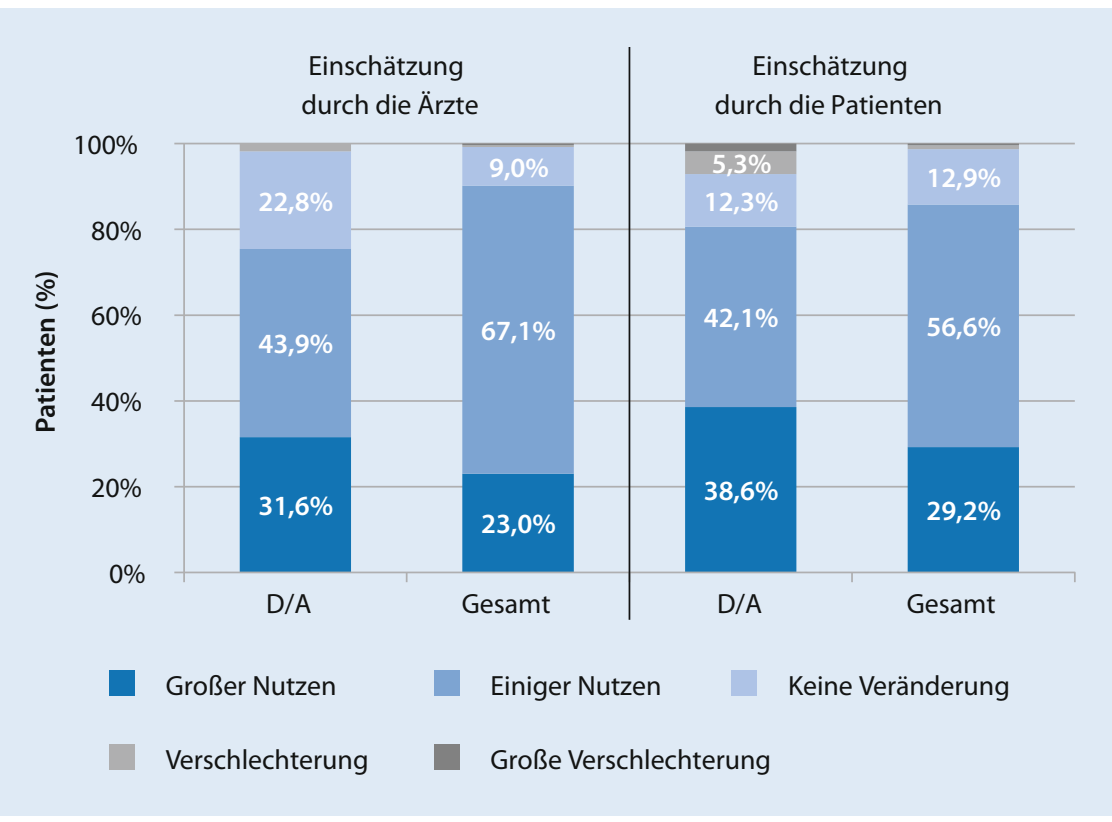

Abb. 4 ॥ Globale Einschätzung des Therapienutzens durch Ärzte und Patienten

weglichkeit im spastischen Bewegungssegment. In der ULIS-II-Studie wiesen die Patienten eine durchschnittliche Erkrankungsdauer von mindestens 5 Jahren, in der deutsch/österreichischen Kohorte sogar von 8 Jahren auf. Dieser zeitliche Unterschied der Erkrankungsdauer und das häufigere Auftreten von Kontrakturen bei der D/A-Kohorte im Vergleich zur Gesamtkohorte sind mögliche Gründe, warum das am meisten genann-
Studie, um eine validierte Aussage treffen zu können.

Die vergleichbar gute Zielerreichung sowohl in der D/A- als auch der Gesamtkohorte spricht für eine sehr gute Zielsetzung. Abweichungen zeigen sich am deutlichsten in der Zielerreichung der Verbesserung aktiver Funktionen, welches in dieser Population mit chronischer Spastizität auch eines der ambitioniertesten Ziele darstellt und dadurch schwieriger zu erreichen ist. Die Aussagekraft für die beiden Ziele Mobilität und unwillkürliche Bewegung ist aufgrund der in beiden Gruppen niedrigsten Gruppengröße am geringsten.

Der GAS-T-Score der deutsch/österreichischen Patientengruppe lag mit 48,1 etwas unter dem der Gesamtkohorte $(52,0)$. Dennoch liegen beide Werte dicht am Erwartungswert $50 \pm 10$ [25], was für eine erfahrene und gute Zusammenarbeit von Patient und Ärzteteam beim Setzen der erreichbaren Ziele spricht. In der Literatur wird die Veränderung des GAS-T-Scores um $>10$ als klinisch relevant beschrieben $[25,27,30]$. Der Anstieg um 14,8 in der D/A-Gruppe bzw. um 17,6 in der Gesamtkohorte bestätigt die Ergebnisse einer randomisierten klinischen Studie [20] und zeigt, dass BoNT-A auch im Praxisalltag klinisch relevante Veränderungen auf funktionaler Ebene herbeiführen kann. Dies wurde auch in einer doppelblinden, randomisierten, kontrollierten Studie anhand der signifikanten Veränderung der aktiven und passiven Funktion bestätigt [15].

Bemerkenswert ist die Reduktion von Begleittherapien zur zweiten Visite, insbesondere der oralen antispastischen Medikation, welche häufig mit Nebenwirkungen assoziiert ist bzw. bei Langzeitanwendung über mehrere Monate infolge einer Toleranzbildung ihre Wirkung verliert [3]. Unter BoNT-A wurden dagegen bei den D/A-Patienten keine unerwünschten Arzneimittelwirkungen beobachtet. Viele Patienten erhielten zum Injektionszeitpunkt weitere Begleittherapien wie Schienen und Lagerung. Eine Abnahme dieser Maßnahmen hat positive Folgen für das Gesundheitswesen und bedeutet weniger Belastung für den Patienten und das Pflegeteam. Ob jedoch die Reduktion in der Notwendigkeit von 
Begleittherapien eine kausale Folge von BoNT-A ist, muss in gut kontrollierten klinischen Studien verifiziert werden. Die Behandlung mit BoNT-A wurde sowohl von Ärzten als auch von Patienten insgesamt sehr positiv bewertet, was für einen hohen Nutzen der Therapie in der täglichen Praxis spricht.

Die Studie ist durch das nichtinterventionelle Studiendesign und das Fehlen einer Kontrollgruppe limitiert, sodass keine kausalen Schlüsse zulässig sind. Die Begrenzung der Patientenzahl pro Zentrum auf 5 bis 12 könnte die Ergebnisse etwas verzerren, da möglicherweise bei der Selektion der Patienten solche mit weniger häufigen Formen der Armspastik unterrepräsentiert waren. Der Vergleich der deutsch/österreichischen Subgruppe mit der Gesamtkohorte ist statistisch vorsichtig zu interpretieren, da die D/A-Patienten in der Gesamtkohorte ebenfalls enthalten sind und 12,5\% dieser Population ausmachen. Die beobachteten Unterschiede zwischen den beiden Gruppen sind demnach in der Realität sogar gröBer als die Zahlen vermuten lassen. In der ULIS-II-Studie wurde nur ein Injektionszyklus über 3 Monate beobachtet, was für die meisten gesetzten Ziele ein relativ kurzer Zeitraum ist. In der oben erwähnten ULIS-III-Studie wird derzeit der Effekt der BoNT-A-Behandlung bei wiederholten Injektionen über 2 Jahre beobachtet. Dadurch erwarten wir noch validere Daten zum Langzeitverlauf der Praxis der BoNT-A-Behandlung.

\section{Fazit für die Praxis}

- Nach einmaliger Injektion von BoNTA wurde eine hohe Responderrate von ca. $80 \%$ erzielt, definiert als das Erreichen des primär gesetzten individuellen Therapieziels.

- Die Mehrheit der Patienten erreichte die gesetzten Therapieziele, insbesondere in Bezug auf passive Funktionen und Schmerz.

- Die Injektion mit BoNT-A führte somit zu Verbesserungen im Lebensalltag von Patienten und Betreuern, die über die Abnahme von Tonus oder Spastizität hinausgehen.

- Die Analyse der D/A-Subgruppe der ULIS-II-Population zeigt, dass die Behandlung von Patienten mit Armspastik nach Schlaganfall in Deutschland und Österreich gut dokumentiert ist und auf qualitativ hohem Niveau stattfindet.

- Trotz guter Ergebnisse, könnte generell die Behandlungsroutine noch optimiert werden, insbesondere die breitflächige interdisziplinäre Zusammenarbeit sowie die langfristige Einbindung der Patienten in multidisziplinäre Rehabilitationsprogramme.

\section{Korrespondenzadresse}

\section{Dr. K. Fheodoroff}

Gailtal-Klinik

Radnigerstraße 12, 9620 Hermagor, Österreich klemens.fheodoroff@me.com

Förderung. Diese Studie wurde von Ipsen Pharma $\mathrm{GmbH}$ finanziell unterstützt.

\section{Einhaltung ethischer Richtlinien}

Interessenkonflikt. K. Fheodoroff war als Referent für die Firma Ipsen, Merz und Allergan tätig und erhielt Beraterhonorare von der Firma Ipsen und Merz. D. Dressler erhielt Honorare für Beratungen von Allergan, Ipsen, Merz, Abbvie, Bayer, IAB Interdisziplinärer Arbeitskreis Bewegungsstörungen, Medtronic, St Jude und Boston Scientific. D. Dressler hält mehrere Patente zu Botulinumtoxinen und Botulinumtoxintherapie. D. Dressler ist Inhaber von Aktien von Allergan Plc. H. Woldag war als Referent und Berater für die Firma Ipsen tätig und erhielt von dieser Firma eine uneingeschränkte Forschungsunterstützung. P. Koßmehl war als Referent für die Firmen Ipsen, Merz und Medtronic tätig und erhielt Beraterhonorare von den Firmen Ipsen, Merz und Medtronic. M. Koch und P. Maisonobe sind Angestellte der Ipsen Pharma GmbH. G. Reichel war als Referent für die Firmen Ipsen, Merz und Allergan tätig.

Alle beschriebenen Untersuchungen am Menschen wurden mit Zustimmung der zuständigen Ethik-Kommission, im Einklang mit nationalem Recht sowie gemäß der Deklaration von Helsinki von 1975 (in der aktuellen, überarbeiteten Fassung) durchgeführt. Von allen beteiligten Patienten liegt eine Einverständniserklärung vor.

Open Access Dieser Artikel wird unter der Creative Commons Namensnennung 4.0 International Lizenz (http://creativecommons.org/licenses/by/4.0/deed. de) veröffentlicht, welche die Nutzung, Vervielfältigung, Bearbeitung, Verbreitung und Wiedergabe in jeglichem Medium und Format erlaubt, sofern Sie den/die ursprünglichen Autor(en) und die Quelle ordnungsgemäßnennen, einen Link zur Creative Commons Lizenz beifügen und angeben, ob Änderungen vorgenommen wurden.

\section{Literatur}

1. Ashford S, Turner-Stokes L (2006) Goal attainment for spasticity management using botulinum toxin. Physiother Res Int 11(1):24-34

2. Baguley IJ, Nott MT, Turner-Stokes L et al (2011) Investigating muscle selection for botulinum toxin-A injections in adults with post-stroke upper limb spasticity. J Rehabil Med 43(11):1032-1037

3. Bakheit AM, Fedorova NV, Skoromets AA et al (2004) The beneficial antispasticity effect of botulinum toxin type $A$ is maintained after repeated treatment cycles. J Neurol Neurosurg Psychiatr 75(11):1558-1561

4. Bakheit AM, Pittock S, Moore AP et al (2001) A randomized, double-blind, placebo-controlled study of the efficacy and safety of botulinum toxin type $A$ in upper limb spasticity in patients with stroke. Eur J Neurol 8(6):559-565

5. Bakheit AM, Thilmann AF, Ward AB et al (2000) A randomized, double-blind, placebo-controlled, dose-ranging study to compare the efficacy and safety of three doses of botulinum toxin type A (Dysport) with placebo in upper limb spasticity after stroke. Stroke 31(10):2402-2406

6. Bakheit AM, Zakine B, Maisonobe P et al (2010) The profile of patients and current practice of treatment of upper limb muscle spasticity with botulinum toxin type A: an international survey. Int JRehabil Res 33(3):199-204

7. Bhakta BB, Cozens JA, Chamberlain MA et al (2000) Impact of botulinum toxin type $A$ on disability and carer burden due to arm spasticity after stroke: a randomised double blind placebo controlled trial. J Neurol Neurosurg Psychiatr 69(2):217-221

8. Blasi J, Chapman ER, Link E et al (1993) Botulinum neurotoxin A selectively cleaves the synaptic protein SNAP-25. Nature 365(6442):160-163

9. Borg J, Ward AB, Wissel J et al (2011) Rationale and design of a multicentre, double-blind, prospective, randomized, European and Canadian study: evaluating patient outcomes and costs of managing adults with post-stroke focal spasticity. JRehabil Med 43(1):15-22

10. Diener H-C, Weimar C (Hrsg) (2012) Leitlinien für Diagnostik und Therapie in der Neurologie. Thieme, Stuttgart, September (Herausgegeben von der Kommission „Leitlinien“ der Deutschen Gesellschaft für Neurologie)

11. Dietz V, Sinkjaer T (2007) Spastic movement disorder: impaired reflex function and altered muscle mechanics. Lancet Neurol 6(8):725-733

12. Eftekhar P, Mochizuki G, Dutta T et al (2016) Goal attainment scaling in individuals with upper limb spasticity post stroke. Occup Ther Int 23(4):379-389

13. Esquenazi $A$, Albanese $A$, Chancellor $M B$ et al (2013) Evidence-based review and assessment of botulinum neurotoxin for the treatment of adult spasticity in the upper motor neuron syndrome. Toxicon 67:115-128

14. Fheodoroff K, Ashford S, Jacinto J et al (2015) Factors influencing goal attainment in patients with post-stroke upper limb spasticity following treatment with botulinum toxin $A$ in real-life clinical practice: sub-analyses from the Upper Limb International Spasticity (ULIS)-IIStudy. Toxins (Basel) 7(4):1192-1205

15. Gracies JM, Brashear A, Jech R et al (2015) Safety and efficacy of abobotulinumtoxinA for hemiparesis in adults with upper limb spasticity after stroke or traumatic brain injury: a doubleblind randomised controlled trial. Lancet Neurol 14(10):992-1001 
16. Gracies JM, O'Dell M, Vecchio M et al (2018) Effects of repeated abobotulinumtoxin $A$ injections in upper limb spasticity. Muscle Nerve 57(2):245-254

17. Hanlan A, Mills P, Lipson R et al (2017) Interdisciplinary spasticity management clinic outcomes using the goal attainment scale: a retrospective chart review. J Rehabil Med 49(5):423-430

18. Kiresuk TJ, Sherman RE (1968) Goal attainment scaling: a general method for evaluating comprehensive community mental health programs. Community Ment Health J 4(6):443-453

19. Marciniak C, McAllister P, Walker H et al (2017) Efficacy and safety of AbobotulinumtoxinA (Dysport) for the treatment of Hemiparesis in adults with upper limb spasticity previously treated with Botulinum toxin: Subanalysis from a phase 3 randomized controlled trial. $\mathrm{Pm} \mathrm{R}$ 9(12):1181-1190

20. McCrory $P$, Turner-Stokes L, Baguley IJ et al (2009) Botulinum toxin A for treatment of upper limb spasticity following stroke: a multi-centre randomized placebo-controlled study of the effects on quality of life and other person-centred outcomes. J Rehabil Med 41(7):536-544

21. Royal College of Physicians, British Society of Rehabilitation Medicine, Chartered Society of Physiotherapy, Association of Chartered Physiotherapists Interested in Neurology (2009) Spasticity in adults: management using botulinum toxin. National guidelines. London: RCP

22. Simpson DM, Hallett M, Ashman EJ et al (2016) Practice guideline update summary: Botulinum neurotoxin for the treatment of blepharospasm, cervical dystonia, adult spasticity, and headache: report of the guideline development subcommittee of the American academy of neurology. Neurology 86(19):1818-1826

23. Smith SJ, Ellis E, White $S$ et al (2000) A doubleblind placebo-controlled study of botulinum toxin in upper limb spasticity after stroke or head injury. Clin Rehabil 14(1):5-13

24. Tennant A (2007) Goal attainment scaling: current methodological challenges. Disabil Rehabil 29(20-21):1583-1588

25. Turner-Stokes L (2009) Goal attainment scaling (GAS) in rehabilitation: a practical guide. Clin Rehabil 23(4):362-370

26. Turner-Stokes L, Ashford S, Jacinto J et al (2016) Impact of integrated upper limb spasticity management including botulinum toxin $A$ on patient-centred goal attainment: rationale and protocol for an international prospective longitudinal cohort study (ULIS-III). BMJ Open 6:e11157. https://doi.org/10.1136/bmjopen2016-011157

27. Turner-Stokes L, Baguley IJ, De Graaff $S$ et al (2010) Goal attainment scaling in the evaluation of treatment of upper limb spasticity with botulinum toxin: a secondary analysis from a double-blind placebo-controlled randomized clinical trial. J Rehabil Med 42(1):81-89

28. Turner-Stokes L, Fheodoroff K, Jacinto J et al (2013) Results from the Upper Limb International Spasticity Study-II (ULISII): a large, international, prospective cohort study investigating practice and goal attainment following treatment with botulinum toxin A in real-life clinical management. BMJOpen 3(6):e2771

29. Turner-Stokes L, FheodoroffK, Jacinto J et al (2013) Upper limb international spasticity study: rationale and protocol for a large, international, multicentre prospective cohort study investigating management and goal attainment following treatment with botulinum toxin A in real-life clinical practice. BMJOpen 3(3):e2230

30. Turner-Stokes L, Williams H, Johnson J (2009) Goa attainment scaling: does it provide added value as a person-centred measure for evaluation of outcome in neurorehabilitation following acquired brain injury? J Rehabil Med 41(7):528-535

31. Welmer AK, Widén Holmqvist L, Sommerfeld DK (2010) Location and severity of spasticity in the first $1-2$ weeks and at 3 and 18 months after stroke. Eur JNeurol 17(5):720-725

32. Yip AM, Gorman MC, Stadnyk K et al (1998) A standardized menu for Goal Attainment Scaling in the care offrailelders. Gerontologist 38(6):735-742
Neurodegeneration Research Award

Der mit 100.000 Euro dotierte „Neurodegeneration Research Award" geht an zwei Neurowissenschaftlerinnen aus München und Boston. Mit dem Preisgeld wollen Sabina Tahirovic, vom Deutschen Zentrum für Neurodegenerative Erkrankungen, und Susan Cotman, vom Massachusetts General Hospital, Gemeinsamkeiten von Demenzerkrankungen bei Kindern und Erwachsenen erforschen.

Im Fokus des auf zwei Jahre ausgelegten Projekts stehen die Immunzellen des Gehirns. Dieser erstmals verliehene Forschungsförderpreis wurde von der NCLStiftung mit Unterstützung der Joachim Herz Stiftung ins Leben gerufen. Zu den sogenannten Kinderdemenzen zählen beispielsweise die "Niemann-Pick Typ C Krankheit" (NPC) und die „Neuronale Ceroid Lipofuszinose" (NCL), von der diverse Varianten bekannt sind. Ausgelöst werden NPC und NCL durch Fehler im Erbgut. Die Folge: in den Nerven- und anderen Hirnzellen sammeln sich schwer abbaubare Substanzen an. An dieser Überfrachtung gehen die Nervenzellen letztlich zugrunde.

\section{Aktive Immunzellen}

"Trotz aller Unterschiede haben diese Erkrankungen eine Reihe von Gemeinsamkeiten mit Alzheimer", erläutert Dr. Tahirovic. „Dazu gehört die Aktivierung der Immunzellen des Gehirns, der sogenannten Mikroglia. Infolgedessen entwickelt sich eine chronische Entzündung. Das Gehirn steht dann gewissermaßen unter Dauerbeschuss."

Genau diese Prozesse wollen Tahirovic und ihre US-amerikanische Kollegin unter die Lupe nehmen.

Quelle: idw-online.de, Dr. Marcus Neitzert (www.dzne.de) 